\title{
Prevalência e fatores associados à presença de anemia em idosos do município de Viçosa (MG), Brasil
}

\author{
Prevalence and factors associated with the presence of anemia \\ in the elderly of the municipality of Viçosa, State of Minas Gerais, \\ Brazil
}

Clarice Santana Milagres ${ }^{1}$

Keila Bacelar Duarte de Moraes ${ }^{2}$

Sylvia Carmo Castro Franceschini ${ }^{2}$

Luciana Ferreira Rocha Sant'Ana ${ }^{2}$

Luciana Moreira Lima ${ }^{3}$

Andréia Queiroz Ribeiro ${ }^{2}$

\footnotetext{
${ }^{1}$ Faculdade de Odontologia de Piracicaba, Universidade Estadual de Campinas. Av. Limeira 901, Vila Rezende. 13414903 Piracicaba SP Brasil.claricemilagres01@ gmail.com

${ }^{2}$ Departamento de Nutrição e Saúde, Universidade Federal de Viçosa (UFV).

${ }^{3}$ Departamento de Medicina e Enfermagem, UFV.
}

\begin{abstract}
Anemia is the most common hematological problem encountered in the elderly population. A cross-sectional, population-based survey was conducted to evaluate the prevalence and factors associated with anemia in the elderly in Viçosa (State of Minas Gerais). Data were collected by means of a household survey and conducting biochemical tests on 349 elderly between June and December 2009. The prevalence of anemia was $11.7 \%$ (95\% CI 8.3\%-15.1\%) and was found to be higher among men (15.4\%) among those aged 80 years and older (30\%) and those who practiced polypharmacy (16.8\%). The results obtained indicate anemia determinants similar to those observed in developed countries. The real need of polypharmacy should be evaluated in health care for the elderly, in order to prevent iatrogenic complications, of which anemia is one such complication.
\end{abstract}

Key words Anemia, Hemoglobin, Determinants, Elderly
Resumo A anemia é o problema hematológico mais comum encontrado na população idosa. Com objetivo de avaliar a prevalência e os fatores associados à anemia em idosos no município de Viçosa (MG), foi realizado um estudo transversal, de base populacional. Os dados foram coletados de junho a dezembro de 2009, mediante inquérito domiciliar e realização de exames bioquímicos em 349 idosos. A prevalência de anemia foi de 11,7\% (IC95\% 8,3\%-15,1\%) e mostrou-se mais elevada entre os homens $(15,4 \%)$, entre idosos com 80 anos e mais $(30,0 \%)$ e naqueles que praticavam polifarmácia (16,8\%). Os resultados evidenciaram determinantes de anemia semelhantes ao observado em países desenvolvidos. A real necessidade da polifarmácia deve ser avaliada na atenção à saúde dos idosos, com vistas a prevenir iatrogenias, dentre as quais a anemia está incluída.

Palavras-chave Anemia, Hemoglobina, Determinantes, Idosos 


\section{Introdução}

O envelhecimento possui relação com o declínio funcional de múltiplos sistemas orgânicos e dessa forma pode aumentar a probabilidade de ocorrência de disfunções e doenças nesses indivíduos ${ }^{1}$. Logo, idosos podem apresentar declínio nas atividades físicas, na capacidade funcional, mudanças dos padrões alimentares, bem como processos inflamatórios. Esses fatores podem refletir no aparecimento de anemia, a qual demonstra ser o problema hematológico mais comumente encontrado nos indivíduos idosos ${ }^{2,3}$.

A anemia no idoso é consequência de três grandes diferentes vertentes de causalidade. Uma delas é a anemia causada por deficiências nutricionais, correspondendo a um terço de todas elas, sendo a deficiência de ferro a principal. Há também a decorrente de doenças crônicas (ou anemia da inflamação crônica e/ou da doença renal crônica) e a inexplicada ${ }^{4-7}$.

No idoso, a anemia é uma condição subdiagnosticada, podendo refletir uma doença de base, como infecções e neoplasias. Condições associadas à anemia estão relacionadas a um declínio nos desempenhos físico e mental e nas habilidades de manutenção das atividades de vida diária e no aumento da fragilidade. Consequentemente, possibilita um aumento na morbimortalidade dessa população ${ }^{1,3,8}$.

Estudos nacionais e internacionais estimam prevalências entre $4 \%$ e $12 \%$ de anemia entre idosos $^{9-15}$. No entanto, ainda são poucos os estudos nacionais de base populacional em municípios de pequeno e médio porte, de forma que conhecer a prevalência de anemia em idosos da comunidade pode subsidiar informações para localidades que possuam características populacionais semelhantes e, principalmente, podem direcionar ações de melhoria no estado hematológico desse grupo.

Nesse contexto, o presente trabalho objetivou determinar a prevalência de anemia e os fatores associados a essa condição em idosos do Município de Viçosa, Minas Gerais, Brasil.

\section{Materiais e métodos}

Estudo de base populacional, observacional e transversal, conduzido no Município de Viçosa, no período de junho a dezembro de 2009. Localizado na região da zona da mata mineira, em 2007 contava com uma população de 70.404 habitantes, dos quais 7.034 tinham 60 anos ou mais de idade ${ }^{16}$. Dentre estes, $55,4 \%$ são do sexo feminino. Com relação à distribuição da faixa etária $53,7 \%$ dos idosos possuem idade entre 60 69 anos e 31,5\% entre 70-79 anos (DATASUS, $2014)^{17}$

O estudo foi aprovado pelo Comitê de Ética em Pesquisa com Seres Humanos da Universidade Federal de Viçosa.

\section{População alvo}

A população alvo deste estudo foi constituída pelos idosos não institucionalizados residentes no Município de Viçosa. Essa população foi recenseada durante a Campanha Nacional de Vacinação do Idoso, no período de abril a maio de 2008. Para identificação de idosos não participantes da campanha de vacinação e complementação da base cadastral, procedeu-se à confrontação e junção desse banco de dados de recenseamento com outros disponíveis no município. Após essa junção dos bancos, o registro de pessoas com 60 anos e mais totalizou 7.980, que serviu de base para a obtenção da amostra. Maiores detalhes em Nascimento et al. ${ }^{18}$.

\section{Cálculo amostral}

O presente estudo integra um projeto maior intitulado "Condições de saúde, nutrição e uso de medicamentos por idosos do município de Viçosa (MG): um estudo de base populacional", o qual tem como objeto de estudo diferentes desfechos em saúde. Para tanto, o cálculo do tamanho amostral considerou um nível de $95 \%$ de confiança, prevalências estimadas de $50 \%$ e erro tolerado de 4\%. Assim, a amostra calculada foi de 558 idosos, à qual se acrescentou $20 \%$ para cobrir possíveis perdas, totalizando 670 pessoas a serem estudadas $^{18}$. Excluídas as perdas, foram entrevistados 621 idosos, dos quais 349 constituíram uma subamostra $(56,1 \%)$ que se submeteu a exames hematológico e bioquímico, sendo que esses correspondem à amostra objeto de análise desse artigo. Para fins do presente artigo, considerou-se somente o exame hematológico. A distribuição da amostra por sexo foi semelhante ao observado para a população idosa do município de Viçosa. Quanto à faixa etária, a amostra é relativamente mais jovem em comparação à população idosa em geral.

\section{Coleta de dados}

As informações foram obtidas usando-se um questionário semiestruturado com a maioria das 
perguntas fechadas e pré-codificadas. $\mathrm{O}$ questionário foi aplicado diretamente ao idoso, mas caso ele expressasse alguma dificuldade o respondente próximo o auxiliava. Cerca de $25,7 \%$ dos idosos foram auxiliados em alguma seção do questionário, sendo a grande maioria dos auxílios prestada por familiares $(95 \%)^{18}$.

O exame hematológico constou de hemograma completo e para sua realização foi coletada uma amostra de $15 \mathrm{~mL}$ de sangue por um técnico do Laboratório de Análises Clínicas da Divisão de Saúde da Universidade Federal de Viçosa.

A mensuração da concentração de hemoglobina foi realizada pelo método de impedância no aparelho Coulter T-890, utilizando-se o kit Beckman Coulter ${ }^{18}$.

\section{Variáveis do estudo}

A variável dependente analisada foi anemia, definida pela Organização Mundial de Saúde (1968) como a mensuração dos níveis de hemoglobina plasmática $<12 \mathrm{~g} / \mathrm{dL}(7,5 \mathrm{mmol} / \mathrm{L})$ para o sexo feminino e $<13 \mathrm{~g} / \mathrm{dL}(8,1 \mathrm{mmol} / \mathrm{L})$ para o sexo masculino ${ }^{3}$.

As variáveis independentes analisadas foram:

a) Características sociodemográficas: sexo (masculino, feminino), idade (60-69 anos, 70-79 e 80 anos e mais), escolaridade (nunca estudou, até quatro anos e cinco anos ou mais) e renda (de acordo com a mediana: maior que $\mathrm{R} \$ 715,00 \mathrm{e}$ menor ou igual a $\mathrm{R} \$ 715,00)$.

b) Indicadores das condições de saúde e uso dos serviços de saúde: história de diabetes, hipertensão arterial, dislipidemias, doença renal, depressão, infarto agudo do miocárdio, número de doenças autorreferidas (até quatro doenças, e cinco ou mais doenças autorrelatadas), capacidade funcional (adequada e inadequada), percepção da própria saúde (muito boa/boa, regular, ruim/muito ruim), estado nutricional (eutrofia, baixo peso e excesso de peso), alteração da ingestão alimentar nos últimos três meses (sem redução e com redução), número de medicamentos consumidos nos últimos 15 dias (até quatro e cinco ou mais), número de consultas médicas nos últimos 12 meses anteriores à entrevista (nenhuma vez, até cinco vezes e seis vezes ou mais), posse de plano privado de saúde e internações hospitalares no último ano (nenhuma e uma vez ou mais).

O estado nutricional foi avaliado pelo índice de massa corporal (IMC), com base na divisão do peso em quilogramas pela estatura em metro elevada ao quadrado $\left(\mathrm{kg} / \mathrm{m}^{2}\right)$. Para tal, os pontos de corte adotados para avaliar o estado nutricional foram os propostos por Lipschitz ${ }^{19}$ : baixo peso $(<$ $\left.22 \mathrm{~kg} / \mathrm{m}^{2}\right)$, eutrofia $\left(22-27 \mathrm{~kg} / \mathrm{m}^{2}\right)$ e sobrepeso $(>$ $\left.27 \mathrm{~kg} / \mathrm{m}^{2}\right)$.

A avaliação da capacidade funcional baseouse no relato dos sujeitos em uma escala de 12 tipos de atividades entre as de vida diária (AVD) e as instrumentais de vida diária (AIVD). As AVD incluídas foram: banhar-se, vestir-se, comer sozinho, ir ao banheiro, caminhar de um cômodo a outro na casa e sair da cama em direção à cadeira. Já as atividades instrumentais de vida diária (AIVD) foram preparar ou cozinhar o alimento, usar o telefone, sair de casa e pegar um ônibus, tomar a medicação, manusear o dinheiro, fazer compras, arrumar a casa, lavar e passar ${ }^{18}$.

Do relato das atividades funcionais, 12 foram analisadas e divididas em categorias: 1) sem dificuldade; 2) com pouca dificuldade; 3 ) com muita dificuldade; 4) não capaz; 5) não se aplica. Subsequentemente essas categorias foram somadas. Para fins de análise estatística, a variável capacidade funcional foi dicotomizada em adequada e inadequada ${ }^{18}$. Assim, os indivíduos que relataram alguma dificuldade em realizar seis ou mais atividades (categorias 2 e 3), ou quando consideraram que, pelo menos, três atividades eram difíceis de executar (categoria 4) foram considerados como tendo a capacidade funcional inadequada ${ }^{18}$.

\section{Análise dos dados}

Para o armazenamento dos dados foi utilizado o software EpiInfo versão 6.04 e para a análise foi empregado o software Stata versão 11.0. A normalidade da distribuição das variáveis quantitativas foi avaliada usando-se o teste de Kolmogorov-Smirnov. Análise descritiva das variáveis foi apresentada por meio de medidas de tendência central e de variabilidade adequadas, bem como distribuição de frequências.

A comparação dos níveis de hemoglobina entre homens e mulheres, estratificada pela faixa etária, foi realizada com o teste t de Student. A associação entre as variáveis independentes categóricas e a presença de anemia na análise bivariada foi avaliada pelo teste qui-quadrado de Pearson $\left(\chi^{2}\right)$. O nível de significância adotado em todas as comparações foi de $\alpha=5 \%$.

Foram realizadas análises bivariadas entre as variáveis dependentes e independentes, obtendose como medida de associação a razão de prevalência (RP) e os respectivos intervalos de confiança de $95 \%$ por meio da regressão de Poisson com variância robusta. Foram incorporadas na análise 
multivariada as variáveis que se associaram com o desfecho, na análise bivariada, com valor de $\mathrm{p}$ $<0,25$ e mantidas no modelo final aquelas com associação significante no nível de $\mathrm{p}<0,05$.

\section{Aspectos éticos}

A coleta de dados foi realizada após a assinatura do termo de consentimento livre e esclarecido (TCLE) pelo idoso ou seu cuidador e o protocolo da pesquisa foi aprovado pelo Comitê de Ética em Pesquisa com Seres Humanos da Universidade Federal de Viçosa.

\section{Resultados}

Dentre os 349 idosos incluídos no estudo, havia equivalência na proporção de homens e mulheres, sendo que as últimas representavam $49,9 \%$. A média da idade foi de 69,6 anos ( dp = 7,51 anos) com a faixa etária de 60 a 69 anos e 70 a 79 anos correspondendo a $57,3 \%$ e $31,2 \%$, respectivamente.

Nessa amostra, encontrou-se nível sérico médio de hemoglobina de 13,7 $\mathrm{g} / \mathrm{dL}(\mathrm{dp}=1,3 \mathrm{~g} / \mathrm{dL})$, correspondente à faixa de normalidade para indivíduos idosos de ambos os sexos. O valor mínimo foi $8,2 \mathrm{~g} / \mathrm{dL}$ enquanto o máximo foi $17,5 \mathrm{~g} / \mathrm{dL}$. Observaram-se diferenças significantes no nível médio de $\mathrm{Hb}$ entre homens e mulheres nas faixas etárias de 60 a 69 anos e 70 a 79 anos. Em ambas as faixas esses níveis médios foram significantemente inferiores nas mulheres em comparação aos homens $(13,34 \mathrm{~g} / \mathrm{dL}$ vs $14,26 \mathrm{~g} / \mathrm{dL}$ na faixa etária de 60 a 69 anos e 13,36 g/dL x 14,44 g/dL entre 70 e 79 anos, respectivamente) (Figura 1).

A prevalência global de anemia foi de 11,7\% (IC95\%, 8,3\%-15,1\%). De acordo com a Tabela 1 , observa-se diferença significante na prevalência de anemia em relação ao sexo, idade e escolaridade dos idosos, sendo maior entre aqueles do sexo masculino, com idade mais avançada e sem escolaridade.

Em relação às comorbidades observaram-se maiores prevalências de hipertensão arterial, dislipidemia e diabetes entre os idosos estudados $(75,0 \%, 58,2 \%$ e $20,9 \%$, respectivamente). A doença renal apresentou baixa prevalência, mas se associou com significância marginal com a anemia. Entre os indivíduos investigados, aqueles que autorreferiram cinco ou mais doenças, apresentaram maior frequência de anemia, sendo o dobro daqueles que referiam possuir até quatro comorbidades. Este resultado foi estatisticamente significante, conforme observado na Tabela 2.

A capacidade funcional inadequada também se associou significantemente à anemia na análise bivariada. Com relação à farmacoterapia, todos os idosos participantes deste estudo faziam uso de algum medicamento. Observou-se uma maior prevalência de anemia nos indivíduos que faziam uso de cinco ou mais medicamentos (Tabela 2).

$\mathrm{Na}$ análise multivariada (Tabela 3), verificouse que os idosos do sexo masculino e na faixa etária de 80 e mais anos apresentaram prevalência de anemia 2,60 e 2,40 vezes maior em relação às idosas e ao grupo com idade entre 60 e 69 anos, respectivamente. A polifarmácia, após ajuste pelas demais variáveis também se manteve independentemente associada à anemia.

As variáveis escolaridade e capacidade funcional, embora não se mantiveram independentemente associadas à anemia para um de $5 \%$, foram mantidas no modelo, pois melhoraram o ajuste do mesmo.

\section{Discussão}

Em nosso estudo, os resultados encontrados em relação aos níveis médios de hemoglobina $(13,74$ g/dL) e a prevalência de anemia nos idosos $(11,7 \%)$ são semelhantes ao trabalho conduzido por Chaves et al. ${ }^{20}$, no qual se encontrou uma média de nível de hemoglobina de $14 \mathrm{~g} / \mathrm{dL}$, entre idosos da comunidade de Baltimore, nos Estados Unidos. No entanto, há poucos trabalhos de base populacional com dados sobre a presença de anemia na população idosa, já que se encontra uma extensa variabilidade nos resultados de redução dos níveis de hemoglobina com o aumento da idade $^{9-15,21,22}$. A prevalência de anemia foi maior entre os homens, a despeito de que os níveis médios de $\mathrm{Hb}$ foram significantemente menores entre as mulheres. Esses achados podem ser devidos, em parte, a diferenças no ponto de corte para classificação da anemia, sendo este para os homens mais sensível em comparação ao das mulheres (13,0 vs. 12,0, respectivamente).

Alguns estudos brasileiros apresentam panoramas da anemia entre idosos: Viçosa (MG), cidade com população de aproximadamente 76 mil habitantes; e Camaragibe (PE), com cerca de 145 mil habitantes ${ }^{13,14}$. Em estudos populacionais, Schaan et al. ${ }^{1}$, investigaram idosos saudáveis numa amostra não probabilística em Gravataí (RS), encontrando 10,2\% com anemia. O mesmo resultado foi encontrado por Santos et al. ${ }^{15}$, que contemplaram a cidade de São Paulo. Já resul- 


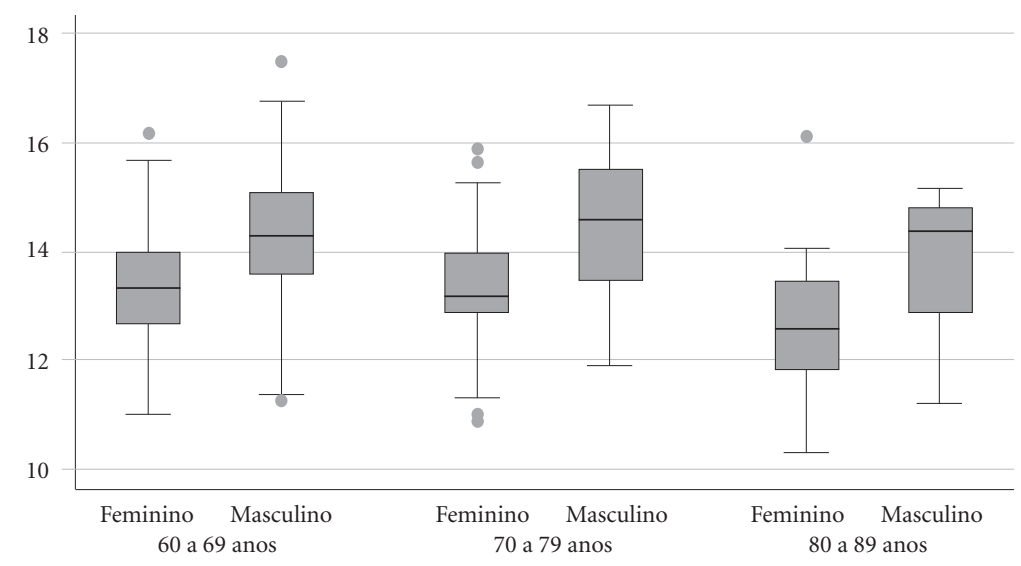

Figura 1. Distribuição dos níveis de hemoglobina entre sexo de acordo com a faixa etária dos idosos de Viçosa, Minas Gerais, 2009.

Tabela 1. Prevalência e razão de prevalência (RP) de anemia, segundo variáveis sociodemográficas em idosos de Viçosa, Minas Gerais, 2009.

\begin{tabular}{|c|c|c|c|c|}
\hline Variáveis/Categorias & $\mathbf{n}^{*}$ & Prevalência (\%) & RP (IC95\%) & Valor $\mathbf{p}^{* *}$ \\
\hline \multicolumn{5}{|l|}{ Sexo } \\
\hline Feminino & 174 & 8,0 & 1,0 & 0,04 \\
\hline Masculino & 175 & 15,4 & $1,92(1,00-3,66)$ & \\
\hline \multicolumn{5}{|l|}{ Faixa etária (anos) } \\
\hline $60-69$ anos & 200 & 8,5 & 1,0 & \\
\hline $70-79$ anos & 109 & 11,0 & $1,29(0,62-2,71)$ & 0,49 \\
\hline 80 anos e mais & 40 & 30,0 & $3,53(1,68-7,39)$ & $<0,01$ \\
\hline \multicolumn{5}{|l|}{ Escolaridade } \\
\hline Nunca estudou & 42 & 23,8 & 1,0 & \\
\hline Até 4 anos & 225 & 10,7 & $0,45(0,21-0,94)$ & 0,03 \\
\hline 5 anos ou mais & 82 & 8,5 & $0,36(0,14-0,94)$ & 0,04 \\
\hline \multicolumn{5}{|l|}{ Renda (mediana) } \\
\hline Maior que $\mathrm{R} \$ 715,00$ & 137 & 16,1 & 1,0 & \\
\hline Menor ou igual a $\mathrm{R} \$ 715,00$ & 178 & 10,1 & $1,58(0,88-2,84)$ & 1,12 \\
\hline
\end{tabular}

*Número de indivíduos na amostra; ** Teste $\chi^{2}$ de Pearson.

tados do projeto Bambuí23 ${ }^{23}$ com dados de 1997, mostraram prevalência de 4,5\% de anemia entre idosos.

O sexo masculino se manteve independentemente associado à presença de anemia. Esse resultado corrobora a literatura, que tem relatado o aumento da prevalência de anemia com a idade em ambos os sexos, sendo este aumento mais acentuado nos homens em comparação às mulheres ${ }^{4}$. Por outro lado, pesquisas observam que a ocorrência de anemia em homens nas idades de 17 a 49 anos é menor em relação às mulheres, ao passo que a partir da quinta década de vida, essa ocorrência modifica-se, passando-se a observar uma menor prevalência de quadro anêmico em mulheres. Alguns autores ressaltam que as diferenças relacionadas ao sexo tendem a diminuir significativamente em pacientes idosos, podendo mesmo chegar a desaparecer. A explicação para isso seria uma diminuição da perda crônica de 
Tabela 2. Prevalência e razão de prevalência (RP) de anemia, segundo variáveis de condição de saúde, nutrição e uso de serviços de saúde em idosos de Viçosa, Minas Gerais, 2009.

\begin{tabular}{|c|c|c|c|c|}
\hline Variáveis/Categorias & $\mathbf{n}^{*}$ & Prevalência (\%) & RP (IC95\%) & Valor $\mathbf{p}^{* *}$ \\
\hline \multicolumn{5}{|l|}{ História de diabetes } \\
\hline Não & 276 & 10,1 & 1,0 & \\
\hline Sim & 73 & 17,8 & $1,75(0,95-3,21)$ & 0,06 \\
\hline \multicolumn{5}{|l|}{ História de hipertensão arterial } \\
\hline Não & 87 & 8,0 & 1,0 & \\
\hline Sim & 262 & 13,0 & $1,61(0,74-3,51)$ & 0,22 \\
\hline \multicolumn{5}{|l|}{ História de dislipidemia } \\
\hline Não & 145 & 10,3 & 1,0 & \\
\hline Sim & 203 & 12,8 & $1,23(0,67-2,25)$ & 0,48 \\
\hline \multicolumn{5}{|l|}{ História de doença renal } \\
\hline Não & 311 & 10,6 & 1,0 & \\
\hline Sim & 38 & 21,1 & $1,98(0,96-3,97)$ & 0,05 \\
\hline \multicolumn{5}{|l|}{ História de depressão } \\
\hline Não & 283 & 12,0 & 1,0 & \\
\hline Sim & 66 & 10,6 & $0,88(0,40-1,90)$ & 0,75 \\
\hline \multicolumn{5}{|c|}{ História de infarto agudo do miocárdio } \\
\hline Não & 326 & 11,7 & 1,0 & \\
\hline Sim & 23 & 13,0 & $1,12(0,37-3,36)$ & 0,84 \\
\hline \multicolumn{5}{|l|}{$\mathrm{N}^{\circ}$ de doenças } \\
\hline Até 4 & 216 & 8,8 & 1,0 & \\
\hline 5 ou mais & 133 & 16,5 & $1,88(1,05-3,34)$ & 0,03 \\
\hline \multicolumn{5}{|l|}{ Capacidade funcional } \\
\hline Adequado & 292 & 9,9 & 1,0 & \\
\hline Inadequado & 56 & 21,4 & $2,15(1,17-3,77)$ & 0,01 \\
\hline \multicolumn{5}{|l|}{ Percepção da saúde } \\
\hline Bom/muito bom & 156 & 10,9 & 1,0 & \\
\hline Regular & 162 & 11,7 & $1,07(0,58-1,99)$ & 0,81 \\
\hline Ruim/muito ruim & 22 & 9,1 & $0,83(0,20-3,37)$ & 0,79 \\
\hline \multicolumn{5}{|l|}{ Estado nutricional } \\
\hline Eutrofia & 137 & 11,7 & 1,0 & \\
\hline Baixo peso & 33 & 21,2 & $0,65(0,31-1,36)$ & 0,25 \\
\hline Excesso de peso & 144 & 7,6 & $1,81(0,81-4,05)$ & 0,14 \\
\hline \multicolumn{5}{|l|}{ Ingestão alimentar } \\
\hline Sem redução & 318 & 11,3 & 1,0 & \\
\hline Com redução & 31 & 16,1 & $1,42(0,60-3,37)$ & 0,42 \\
\hline \multicolumn{5}{|l|}{$\mathrm{N}^{\circ}$ de medicamentos utilizados } \\
\hline Até 4 & 230 & 9,1 & 1,0 & \\
\hline 5 ou mais & 119 & 16,8 & $1,84(1,03-3,26)$ & 0,03 \\
\hline \multicolumn{5}{|l|}{ Consultas médicas } \\
\hline Nenhuma vez & 24 & 8,3 & 1,0 & \\
\hline Até 5 vezes & 268 & 10,4 & $1,25(0,31-4,95)$ & 0,74 \\
\hline 6 vezes ou mais & 56 & 19,6 & $2,35(0,56-9,85)$ & 0,24 \\
\hline \multicolumn{5}{|l|}{ Plano de saúde } \\
\hline Não & 147 & 15,0 & 1,0 & \\
\hline Sim & 201 & 9,5 & $0,63(0,35-1,12)$ & 0,12 \\
\hline \multicolumn{5}{|l|}{ Hospitalizações } \\
\hline Nenhuma & 303 & 10,6 & 1,0 & \\
\hline $1 \mathrm{vez}$ ou mais & 45 & 20,0 & $1,89(0,96-3,70)$ & 0,06 \\
\hline
\end{tabular}

*Número de indivíduos na amostra; * Teste $\chi^{2}$ de Pearson. 
Tabela 3. Resultados finais da análise multivariada da associação entre anemia e variáveis sociodemográficas, condição de saúde, nutrição em idosos de Viçosa, Minas Gerais, 2009.

\begin{tabular}{|c|c|c|}
\hline Variáveis/Categorias & $\begin{array}{l}\text { RP Ajustada } \\
\quad(\text { IC 95\%) }\end{array}$ & Valor $\mathbf{p}^{* *}$ \\
\hline \multicolumn{3}{|l|}{ Sexo } \\
\hline Feminino & 1,0 & \\
\hline Masculino & $2,60(1,43-4,72)$ & $<0,01$ \\
\hline \multicolumn{3}{|l|}{ Faixa etária (anos) } \\
\hline $60-69$ anos & 1,0 & \\
\hline $70-79$ anos & $1,25(0,62-2,53)$ & 0,54 \\
\hline 80 anos e mais & $2,40(1,22-4,73)$ & 0,01 \\
\hline \multicolumn{3}{|l|}{ Escolaridade } \\
\hline Nunca estudou & 1,0 & \\
\hline Até 4 anos & $0,54(0,28-1,04)$ & 0,07 \\
\hline 5 anos ou mais & $0,44(0,18-1,08)$ & 0,07 \\
\hline \multicolumn{3}{|l|}{$\mathrm{N}^{\circ}$ de medicamentos } \\
\hline Até 4 & 1,0 & \\
\hline 5 ou mais & $1,78(1,03-3,07)$ & 0,03 \\
\hline \multicolumn{3}{|l|}{ Capacidade funcional } \\
\hline Adequada & 1,0 & \\
\hline Inadequada & $1,74(0,93-3,25)$ & 0,08 \\
\hline
\end{tabular}

sangue em mulheres devido à menstruação e gravidez. Logo, critérios diferenciados entre homens e mulheres no climatério (há mais de 10 anos) no diagnóstico de anemia não seriam necessários ${ }^{4,7}$.

Já é conhecido que o aumento da idade traz consigo implicações à saúde dos idosos, e isso também foi observado em relação ao aumento na prevalência de anemia (30\% para aqueles com 80 e mais anos). Esses achados corroboram outros trabalhos que identificam maiores taxas de morbimortalidade, maiores comprometimentos no desempenho físico e mental e menores habilidades de manutenção das atividades de vida diária entre idosos mais velhos. O idoso mais velho, com um quadro anêmico, apresenta internações hospitalares mais frequentes, com tempo de ocupação do leito maior se comparado à faixa etária inferior. Em termos de utilização dos serviços de saúde, há maiores problemas de longa duração, podendo exigir intervenções custosas, com tecnologia complexa, a fim de realizar um cuidado adequado. Logo, a atenção dispensada nestes cuidados deverá ser especial, uma vez que a instalação da anemia nestes indivíduos pode ser rápida ${ }^{7,9,17}$.

Observou-se um quadro de doenças crônicodegenerativas nestes idosos anêmicos, no entanto, apenas a doença renal mostrou-se significan- temente associada à anemia na análise bivariada neste estudo. Essa informação vai ao encontro do diagnóstico de anemia, visto que essa condição hematológica pode ser consequência da doença renal e implicar em maiores possibilidades de complicações associadas à doença e também um prejuízo na produção de eritropoietina ${ }^{24}$. Segundo Guralnik et al. ${ }^{7}, 8 \%$ dos participantes mais velhos com anemia e doença renal tiveram depuração insuficiente de creatinina (inferior a $30 \mathrm{~mL} /$ min), enquanto $4 \%$ tiveram anemia causada por insuficiência renal junto ao processo inflamatório $^{5,7}$. A doença renal não se manteve independentemente associada à anemia neste estudo, o que pode ser devido, em parte ao pequeno tamanho amostral, insuficiente para demonstrar essa relação. Estudos adicionais podem elucidar essa relação. Acrescenta-se, ainda, que o sexo masculino é fator de risco para a ocorrência da doença renal e esses achados da associação entre doença renal e anemia em relação ao sexo podem ser diferenciados.

Em relação à farmacoterapia, observou-se o uso de medicamentos em todos os participantes do estudo e uma prevalência do uso de cinco ou mais unidades, caracterizando a polifarmácia em $34,1 \%$. Sabe-se que a prescrição medicamentosa deve considerar a idade, a condição socioeconômica, a escolaridade, a carga de doenças, evitando o número excessivo de fármacos, principalmente de mesmo princípio ativo ${ }^{24,25}$. Sabe-se que o uso de múltiplos medicamentos pode predispor o idoso a não adesão, bem como ao uso incorreto dos mesmos. Esse fato pode comprometer, por exemplo, a terapia para um quadro de anemia já instalado entre idosos do presente estudo, embora afirmativas a esse respeito requeiram uma investigação aprofundada da adesão entre os pacientes. $\mathrm{O}$ uso de algumas classes terapêuticas podem resultar em anemia como reação adversa, por diferentes mecanismos, tais como redução da eritropoiese (quimioterápicos), inibição da absorção de ferro (antiácidos a base de cálcio), interferência no metabolismo do ácido fólico (anticonvulsivantes), entre outros ${ }^{24,25}$. Além disso, o uso de múltiplos medicamentos tem sido associado ao uso de especialidades potencialmente inadequadas, o que pode levar a ocorrência de reações adversas, provocando graves complicações e até mesmo intervenções desnecessárias $^{26}$. Dentre tais iatrogenias, inclui-se a anemia. Acredita-se que neste cenário a atenção primária à saúde tenha papel fundamental através de estratégias que orientem esta população quanto à correta utilização de medicamentos, bem como 
promovam ações voltadas para a prescrição racional de medicamentos ${ }^{26}$.

O presente estudo apresenta limitações. A etnia não foi investigada. Consta na literatura diferenças estatisticamente significantes entre elas, em que afro-americanos possuem uma prevalência três vezes maior de serem acometidos pela anemia se comparados aos indivíduos caucasianos da mesma idade ${ }^{4}$. No mais, ressalta-se que no país ocorre intensa miscigenação étnica, dificultando a análise deste dado. Além disso, o delineamento transversal do presente estudo não permite clarificar as relações temporais de causa e efeito entre a anemia e os fatores considerados. Outra limitação do estudo foi a impossibilidade de se avaliar as principais etiologias da anemia e as classes medicamentosas envolvidas na terapêutica. A despeito dessas limitações, foi possível identificar associações relevantes entre anemia e fatores envolvidos no mecanismo de surgimento e/ou manutenção dessa comorbidade.

\section{Considerações finais}

Encontrou-se uma prevalência de anemia de $11,7 \%$ no grupo de idosos estudados e níveis médios de hemoglobina de 13,74 g/dL. Verificou-se associação entre anemia e sexo masculino, idade avançada e uso de cinco ou mais medicamentos, de forma que idosos que apresentam essas características constituem grupos prioritários de intervenções para prevenir e/ou minimizar a ocorrência desse problema hematológico.

Os resultados obtidos neste trabalho associados à sua característica transversal não permitem clarificar a causa e o efeito entre a anemia e os fatores encontrados. No entanto, contribuem para a identificação de determinantes desse problema hematológico entre idosos brasileiros, tendo em vista a escassez de estudos de base populacional no país. Ainda que a anemia seja uma condição comum nos idosos, seu significado clínico é bastante variável, tendo em vista a multiplicidade de fatores que podem interagir entre si na causalidade dessa doença. Um importante passo na promoção de um envelhecimento saudável reside em identificar adequadamente aqueles idosos que necessitam de cuidados. Isso deve ser baseado em critérios diagnósticos corretos e confiáveis, objetivando evitar sobrecarregar os sistemas de saúde, em especial em países em desenvolvimento, como o Brasil.

Por fim, acredita-se que a efetiva estruturação dos programas que visem à promoção de saúde para idosos é condição potencialmente positiva para evitar ou mesmo adiar o aparecimento da anemia e de doenças ligadas ao envelhecimento.

\section{Colaboradores}

CS Milagres contribuiu na concepção, delineamento, análise e interpretação dos dados, redação do artigo e revisão crítica; KBD Morais contribuiu na concepção e redação do artigo; SCC Franceschini contribuiu na concepção, delineamento e revisão crítica; LFR Sant'Ana e LM Lima contribuíram na concepção e revisão crítica; AQ Ribeiro contribuiu na concepção, delineamento, análise e interpretação dos dados, redação do artigo, revisão crítica e aprovação da versão submetida do artigo. 


\section{Referências}

1. Schaan MDA, Schwanke CHA, Bauer M, Luz C, Cruz IM. Hematological and nutricional parameters in apparently healthy elderly individuals. Rev. Bras. Hematol. Hemoter 2007; 29(2):136-143.

2. Barbosa DL, Arruda IKG, Diniz AS. Prevalência e caracterização da anemia do programa de saúde da família. Rev. Bras. Hematol. Hemoter. 2006; 28(4):288-292.

3. World Health Organization. Nutricional anemia: report of WHO Scientific Group. Geneva: World Health Organization; 1968.

4. Patel KV. Epidemiology of anemia in older adults. Semin Hematol 2008; 45(4):210-217.

5. Guralnik JM, Ershler WB, Schrier SL, Picozzi VJ. Anemia in the elderly: a public health crisis in hematology. Hematology Am Soc Hematol Educ Program 2005:528532.

6. Hoobler I, Hunscher H. Hemoglobin differences between blacks and whites. Am J Clin Nutr 1977; 30(12):1935-1937.

7. Guralnik JM, Eisenstaedt RS, Ferrucci L, Klein HG, Woodman RC. Prevalence of anemia in persons 65 years and older in the United States: evidence for a hight rate of unexplained anemia. Blood 2004; 104(8):2263-2268.

8. Vanasse GJ, Berliner N. Anemia in elderly patients: an emerging problem for the 21st century. Hematology Am Soc Hematol Educ Program 2010:271-275.

9. Tettamanti M, Lucca U, Gandini F, Recchia A, Mosconi P, Apolone G, Nobili A, Tallone MV, Detoma P, Giacomin A, Clerico M, Tempia P, Savoia L, Fasolo G, Ponchio L, Della Porta MG, Riva E. Prevalence, incidence and types of mild anemia in the elderly: the "Health and Anemia" Population-Based Study. Haematologica 2010; 95(11):1849-1856.

10. Carmel R. Anemia and aging: an overview of clinical, diagnostic and biological issues. Blood 2001; 15(1):918.

11. Nilsson-Ehle H, Jagenburg R, Landahl S, Svanborg A. Blood haemoglobin declines in the elderly: implications of reference intervals from age 70 to 88 . Eur J Haemotol 2000; 65(5):297-305.

12. Patel KV, Guralnik JM. Prognostic implications of anemia in older adults. Hematology 2009; 94(1);1-2.

13. Barbosa DL, Arruda HKG, Diniz AS. Prevalência e caracterização da anemia em idosos do Programa de Saúde da Família. Rev Bras Hematol Hemoter 2006; 28(4):288-292.

14. Silva CLA. Fatores associados ao estado nutricional e ao nível de hemoglobina em idosos: Programa de Saúde da Família, Viçosa-MG [dissertação]. Viçosa: Universidade Federal de Viçosa; 2008.

15. Santos IS, Scazufca M, Lotufo PA, Menezes PR, Benseñor IM. Anemia and dementia among the elderly: the São Paulo Ageing \& Health Study. Int Psychogeriatr 2012; 24(1):74-81.
16. Instituto Brasileiro de Geografia e Estatística (IBGE). Projeção da População do Brasil por cidade. [acessado 2013 fev 25]. Disponível em: http://www.ibge.gov.br/ cidadesat/link.php? codmun $=260345$

17. Brasil, Ministério da Saúde (MS). Banco de dados do Sistema Único de Saúde-DATASUS. [acessado 2014 set 29]. Disponível em http://www.datasus.gov.br

18. Nascimento CM, Ribeiro AQ, Cotta RMM, Acurcio FA, Peixoto SV, Priore SE, Franceschini SCC. Estado nutricional e fatores associados em idosos do município de Viçosa, Minas Gerais, Brasil. Cad Saude Publica 2011; 27(12):2409-2418

19. World Health Organization (WHO). Physical status: the use and interpretation of anthropometry. Geneva: WHO; 1995. (Technical Re-port Series, 854).

20. Chaves PHM, Xue QL, Guralnik JM, Ferrucci L, Volpato S, Fried LP. What constitutes normal hemoglobin concentration in community-dwelling disabled older women? J Am Geriatr Soc 2004; 52(11):1811-1816.

21. Francisco PMSB, Belan AP, Barros MBA, Carandina L, Alves MCGP, Goldbaum M, Cesar CLG. Diabetes auto-referido em idosos: prevalência, fatores associados e práticas de controle. Cad Saude Publica 2010; 26(1):175-184.

22. Santos IS, Scazufca M, Lotufo PA, Menezes PR, Benseñor IM. Anemia and dementia among the elderly: the São Paulo Ageing \& Health Study. Int Psychogeriatr 2012; 24(1):74-81.

23. Silva CLA, Lima-Costa MF, Firmo JOA, Peixoto SV. Hemoglobin level in older adults and the association with nutritional status and use of health services: the Bambuí Project. Cad Saude Publica 2012; 28(11):2085-2094.

24. Astor BC, Muntner P, Levin A, Eustace JA, Coresh J. Association of kidney function with anemia: the Third National Health and Nutition Examination Survey (1988-1994). Arch Intern Med 2005; 165(12):2222-2227.

25. Korolkovas A. Dicionário Terapêutico Guanabara 2014/2015. Rio de Janeiro: Guanabara; 2014.

26. Silva AL, Ribeiro AQ, Klein CH, Acurcio FA. Utilização de medicamentos por idosos brasileiros, de acordo com a faixa etária: um inquérito postal. Cad Saude Publica 2012; 28(6):1033-1045.

Artigo apresentado em 05/07/2014

Aprovado em 18/03/2015

Versão final apresentada em 20/03/2015 
